\title{
Estudo das malformações congênitas do aparelho urinário: análise de 6.245 necropsias pediátricas
}

\author{
Study of urinary tract congenital malformations: analysis of 6,245 pediatric autopsies
}

\author{
Lucia de Noronha ${ }^{1}$ \\ Adriane Reichert ${ }^{2}$ \\ Vanessa Dello Monaco Martins ${ }^{2}$ \\ Gilberto Antunes Sampaio ${ }^{3}$ \\ Israil Cat ${ }^{4}$ \\ Maria José Serapião 5
}

\begin{tabular}{|c|c|}
\hline os & re \\
\hline $\begin{array}{l}\text { Malformações } \\
\text { Rim } \\
\text { Necropsia }\end{array}$ & $\begin{array}{l}\text { O presente estudo tece investigações sobre os aspectos epidemiológicos de malformações do aparelho } \\
\text { urinário de uma população de natimortos, neomortos, lactentes, pré-escolares e escolares de uma } \\
\text { amostra representativa da população de Curitiba e região metropolitana - análise de } 40 \text { anos de } \\
\text { necropsias na Unidade de Patologia Pediátrica e Perinatal (UPPP) do Serviço de Anatomia Patológica do } \\
\text { Hospital de Clínicas de Curitiba da Universidade Federal do Paraná (UFPR). Variáveis como sexo, idade, } \\
\text { faixa etária e causas de morte são correlacionadas, estratificando a amostra em grupos específicos de } \\
\text { indivíduos, apontando anormalidades epidemiológicas, doenças raras e a relação das doenças com o } \\
\text { processo de morte. Foram encontrados } 182 \text { casos com malformações do aparelho urinário, } \\
\text { correspondendo a } 2,9 \% \text { do número de necropsias avaliadas. Não foram observadas diferenças entre os } \\
\text { sexos, e houve maior prevalência de recém-natos com tais anormalidades. A classe mais prevalente foi a } \\
\text { de malformações renais e de trato urinário superior, com } 150 \text { casos. }\end{array}$ \\
\hline
\end{tabular}

abstract

The presented study investigates epidemiologic aspects regarding congenital malformations of the urinary tract in a representative sample of stillborns, newborns, infants and children from Curitiba, analising autopsies from the Pediatric and Perinatal Pathology Unit (Service of the Clinical Hospital - Curitiba,

Paraná) in a 40-year period. Sex, age groups and death causes are correlated, dividing the cases in specific groups of study, revealing epidemiologic abnormalities, rare diseases and diseases related to the death process. Out of 6,245 autopsies, 182 cases (2.9\%) presented urinary tract congenital malformations. There was no difference between sex, and the group of newborns was the most prevalent. The group of malformations of the kidney and upper urinary tract contributed with 150 abnormalities.

\section{Introdução}

O aparelho urinário é o terceiro sistema mais afetado por malformações congênitas, precedido pelo sistema nervoso central e pelo sistema cardiovascular $(6,15)$. As malformações podem variar desde anomalias de pouca importância clínica até alterações dismórficas severas e potencialmente letais, tal como agenesia renal.

A prevalência das malformações congênitas urinárias relatadas na literatura pode chegar até $10 \%$ dos nascimentos (13). Estas anormalidades renais ou do trato urinário inferior, tanto quanto suas complicações, são importantes causas de morte durante a infância (18).

Este trabalho tem por objetivo estudar a prevalência e as características anatomopatológicas das malformações do aparelho urinário nas necropsias pediátricas realizadas no Serviço de Anatomia Patológica do Hospital de Clínicas da UFPR no período de 1960 a 2000 (17).
1. Professora adjunta do Departamento de Patologia Médica da Universidade Federal do Paraná (UFPR): responsável pela Unidade de Patologia Pediátrica e Perinatal (UPPP); doutora em Patologia. 2. Acadêmica de Medicina; estagiária da UPPP.

3. Professor auxiliar do Departamento de Patologia Médica da UFPR.

4. Professor titular do Departamento de Pediatria da UFPR.

5. Professora convidada do Departamento de Patologia Médica da UFPR.

Unidade de Patologia Pediátrica e Perinatal do Serviço de Anatomia Patológica (SAP/UFPR). 


\section{Metodologia}

\section{Casuística}

O estudo baseou-se na análise de 6.245 laudos de necropsias perinatais e pediátricas realizadas no Serviço de Anatomia Patológica do Hospital de Clínicas da UFPR no período de 40 anos, nos quais foram encontradas 182 malformações do sistema urinário.

A idade mínima considerada para este estudo foi de 22 semanas de gestação, e a máxima, de 14 anos (1). Todos os pedidos de necropsia foram preenchidos com dados como nome, sexo, idade e história clínica completa.

\section{Método}

Em todos os casos foram realizadas necropsias completas com adequado exame do sistema urinário.

Os casos analisados foram divididos em grupos etários conforme classificação baseada em Nelson (1):

- natimortos (NM): aqueles que tiveram morte intrauterina;

- neomortos ou recém-natos (RN): aqueles que nasceram vivos independente da idade gestacional e que foram a óbito em qualquer período até o $28^{\circ}$ dia de vida, prematuros ou não;

- lactentes (L): crianças que foram a óbito no período compreendido entre o 29ㅇa de vida e 11 meses de idade;

- pré-escolares (PE): crianças que foram a óbito entre 1 ano e 6 anos e 11 meses de idade;

- escolares (E): crianças que foram a óbito entre 7 e 14 anos e 11 meses de idade.

Foram examinados todos os 6.245 laudos de necropsias perinatais e pediátricas do Serviço de Anatomia Patológica em 40 anos, a partir dos quais foram retirados dados como sexo, idade, classificação da malformação urinária e sua relação com o processo de morte.

Quanto ao sexo, foram utilizados três grupos principais para a classificação das crianças: o masculino, o feminino e o intersexo. A idade foi anotada em semanas de gestação para os natimortos; em minutos, horas ou dias para os neomortos; em meses para os lactentes; e em anos para os pré-escolares e escolares.

A classificação das malformações urinárias obedeceu ao padrão proposto por Guilbert e Potter (Quadro) (6).

Após análise de todos os dados obtidos na necropsia, classificou-se a relação da malformação congênita do aparelho urinário com o óbito:
- malformações do aparelho urinário diretamente relacionadas com o óbito: aquelas que tiveram atuação significativa no processo do óbito, podendo ou não estar associadas a alterações sistêmicas ou maternas;

- malformações do aparelho urinário parcialmente relacionadas com o óbito: as malformações que faziam parte de síndrome congênita ou associavam-se a malformações de outros órgãos que, em conjunto, causaram o óbito;

- malformações do aparelho urinário com pouca atuação no óbito: aquelas que foram pouco significativas para o óbito.

As malformações do aparelho urinário foram avaliadas em relação a sexo, grupo etário, desenvolvimento embriológico, seqüência malformativa, malformações de outros órgãos e sistemas, síndromes congênitas e atuação no processo de óbito.

\section{Resultados}

Dos 6.245 casos que compõem o banco de necropsias no período de 1960 a 2000, 21\% apresentaram malformações gerais e 2,9\% do total de casos possuíam malformações do aparelho urinário (MFAU) - 182 casos (Figura 1). Destes, 33 pertencentes ao grupo de recémnatos, 96 ao grupo de natimortos e 53 casos de lactentes, pré-escolares e escolares.

A proporção de casos masculinos com MFAU foi de 54\%, e de femininos, $46 \%(p<0,05)$, como demonstra a Tabela 1.

As malformações que tiveram participação direta na causa do óbito correspondem a 29,6\%; as que contribuíram parcialmente para o óbito constituem 14,8\%, e 55\% tiveram participação pouco significativa para o óbito (Figura 2).

Segundo a classificação proposta por Potter, a maioria das malformações $(n=138)$ pertencia à classe A - malformações renais e do trato urinário superior. A classe B - diferenciação renal anormal - contou com 24 casos; a C - doença cística renal, com 7; e a classe D - malformações do trato urinário inferior, com 36 casos, como mostra a Tabela 2. Como alguns casos de necropsia foram classificados em dois ou mais grupos, o valor total do número de malformações do aparelho urinário divididas em classes foi de 205. Há 20 casos com mais de uma malformação em diferentes classes.

O grupo dos natimortos foi o que mais contribuiu para o número de malformações das quatro classes: $54,34 \%$ 
Quadro

Classe

A. Malformações renais e de trato urinário

\section{Classificação das malformações urinárias segundo Guilbert e Potter}

Subclasse

A1. Anormalidades de massa renal

A2. Anormalidades de forma e posição renal

A3. Anormalidades congênitas do trato urinário superior
- Agenesia renal

- Hipoplasia renal Simples

Oligonefrônica

- Nefromegalia

- Rim supranumerário

- Ectopia e má rotação

- Fusão renal

- Duplicação dos ureteres e da pelve renal

- Obstrução Obstrução infundibular caliceal

Hidronefrose congênita

Obstrução ureteral congênita

Dilatação ureteral

\section{B. Diferenciação}

renal anormal
B1. Aplasia renal e displasia multicística

B2. Displasia hipoplástica

B3. Displasia renal obstrutiva

B4. Displasia cística renal difusa

B5. Disgenesia tubular renal e nefropatias renais induzidas por drogas

\section{Doença cística renal}

C1. Doença policística infantil

C2. Doença policística do adulto

C3. Doença glomerulocística

C4. Doença cística localizada

C5. Cistos renais associados com síndromes de malformações múltiplas

C6. Doença cística medular

C7. Cistos renais multiloculares

\section{Malformações do trato urinário inferior}

- Agenesia e hipoplasia

- Divisão congênita Duplicação da bexiga

- Megabexiga completa Septação

- Anomalias do úraco

- Extrofia de bexiga

D2. Epispádias

D3. Anomalias do trato urinário associadas com ânus imperfurado

D4. Anormalidades congênitas da uretra - Válvula de uretra posterior

- Hipospádia

- Cisto de utrículo prostático

- Pólipo de Verumontanum

- Divertículo e valva uretral anterior

- Duplicação uretral 


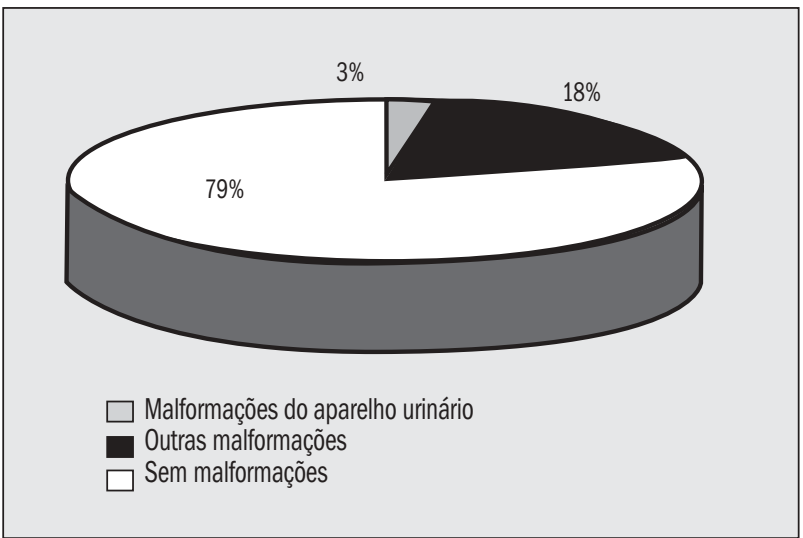

Figura 1 - Relação entre o número de casos de malformações congênitas do aparelho urinário e o número total de casos do banco de necropsias, de 1960 a 2000

dos casos da classe A, $66,66 \%$ da classe B, $42,85 \%$ da classe $C$ e $58,33 \%$ dos casos da classe D.

Quando subdivididas em subclasses, somaram-se 216 malformações. Havia 13 casos que possuíam mais de uma malformação na mesma classe.

Conforme mostra a Tabela 3, a subclasse A3 (anormalidades congênitas do trato urinário superior) foi a que

Tabela 1 aparelho urinário (MFAU)

\section{Proporção do número de casos masculinos e femininos em relação ao número total de casos (banco geral), ao número total de malformações gerais (MF gerais) e ao número de malformações do}

\begin{tabular}{lccccc}
\hline & Banco geral & MF gerais & MFAU & p1 & p2 \\
Masculinos & $54 \%$ & $55 \%$ & $54 \%$ & NS & NS \\
Femininos & $46 \%$ & $45 \%$ & $46 \%$ & NS & NS
\end{tabular}

p1: comparação estatística entre o número de casos do banco geral e de casos de malformações do aparelho urinário; p2: comparação estatística entre 0 número de casos de malformações gerais e de casos de malformações do aparelho urinário; NS: valores sem diferença estatística significativa $(p<0,05)$.

Tabela 2 mação por grupos etários

\begin{tabular}{lcccc} 
& A & B & C & D \\
RN & 21 & 5 & 2 & 6 \\
NM & 75 & 16 & 3 & 21 \\
L/ PE/ E & 42 & 3 & 2 & 9 \\
Total & $\mathbf{1 3 8}$ & $\mathbf{2 4}$ & $\mathbf{7}$ & $\mathbf{3 6}$ \\
\hline
\end{tabular}

RN: recém-natos; NM: natimortos; L/PE/E: lactentes, pré-escolares, escolares; A: malformações renais e de trato urinário superior; $B$ : diferenciação renal anormal; C: doença cística renal; D: malformações do trato urinário inferior.



Figura 2 - Representatividade da malformação congênita do aparelho urinário como causa direta, parcial ou pouco significativa do óbito, de 1960 a 2000

apresentou maior número de casos $(n=63)$, e houve praticamente o mesmo número de casos de anormalidade de massa renal (A1, $n=43)$ e anormalidades de forma e posição renal $(A 2, n=44)$. No grupo $B$ foram encontrados 20 casos de aplasia renal, displasia multicística (B1), um caso de displasia hipoplástica e três casos de displasia cística renal difusa. A classe $\mathrm{C}$ foi a que apresentou menor número de casos $(n=7)$, sendo quatro casos de doença policística infantil (C1), um caso de doença glomerulocística (C3) e dois casos de doença cística localizada (C4). Dos 35 casos classificados como D, 60\% se enquadram na subclasse D4 - anormalidades congênitas da uretra $(n=21)$; dez casos, nas anormalidades congênitas da bexiga (D3); quatro anomalias do trato urinário associadas a ânus imperfurado (D3); e nenhum caso de epispádias.

\section{Discussão}

Malformações dos rins e do trato urinário baixo são causas comuns de falência renal em crianças pequenas, mas pouco se sabe sobre a patogênese molecular destes distúrbios. Em experimentos animais, as principais causas de malformações são mutações, teratógenos químicos e farmacêuticos, obstrução do fluxo urinário fetal e alterações na nutrição materna. Observou-se a possibilidade de associação entre o tabagismo materno durante a gestação e o desenvolvimento de malformações renais, excluindo a doença policística renal geneticamente transmitida, o principal subgrupo das malformações renais $(10,12)$. Em alguns indivíduos com malformações isoladas do trato urinário, a patogênese genética é fortemente sugerida pela história familiar positiva e por estudos de conexão genética, como, por exemplo, o refluxo vesicoureteral. Além disso, malformações esporádicas têm sido demons- 


\section{Tabela 3 Classificação das subclasses malformativas por grupos etários}

\begin{tabular}{|c|c|c|c|c|c|c|c|c|c|c|c|c|c|c|c|c|c|c|c|}
\hline \multirow[t]{2}{*}{ Grupo } & \multicolumn{4}{|c|}{$A=150$} & \multicolumn{4}{|c|}{$B=24$} & \multicolumn{7}{|c|}{$C=7$} & \multicolumn{4}{|c|}{$D=35$} \\
\hline & Al & $\mathrm{A} 2$ & A3 & B1 & B2 & B3 & B4 & B5 & $\mathrm{Cl}$ & $\mathrm{C} 2$ & C3 & C4 & C5 & $\mathrm{C} 6$ & C7 & D1 & D2 & D3 & D4 \\
\hline RN & 6 & 7 & 10 & 2 & - & - & 3 & - & 2 & - & - & - & - & - & - & 1 & - & - & 4 \\
\hline NM & 26 & 19 & 35 & 15 & 1 & - & - & - & 2 & - & 1 & - & - & - & - & 7 & - & 1 & 13 \\
\hline L/PE/E & 11 & 18 & 18 & 3 & - & - & - & - & - & - & - & 2 & - & - & - & 2 & - & 3 & 4 \\
\hline Total & 43 & 44 & 63 & 20 & 1 & - & 3 & - & 4 & - & 1 & 2 & - & - & - & 10 & - & 4 & 21 \\
\hline
\end{tabular}

RN: recém-natos; NM: natimortos; L/PE/E: lactentes, pré-escolares, escolares; A: malformações renais e de trato urinário superior; B: diferenciação renal anormal; C: doença cística renal; D: malformações do trato urinário inferior; A1: anormalidades de massa renal; A2: anormalidades de forma e posição renal; A3: anormalidades congênitas do trato urinário superior; B1: aplasia renal e displasia multicística; B2: displasia hipoplástica; B3: displasia renal obstrutiva; B4: displasia cística renal difusa; B5: disgenesia tubular renal e nefropatias renais induzidas por drogas; C1: doença policística infantil; C2: doença policística do adulto; C3: doença glomerulocística; C4: doença cística localizada; C5: cistos renais associados com síndromes de malformações múltiplas; C6: doença cística medular; C7: cistos renais multiloculares;

D1: anormalidades congênitas da bexiga; D2: epispádias; D3: anomalias do trato urinário associadas com ânus imperfurado; D4: anormalidades congênitas da uretra.

tradas em associação com polimorfismos de genes expressados durante a formação do trato urinário (22).

Aproximadamente um terço de todas as malformações congênitas é encontrado no sistema urogenital (18). A grande maioria das anormalidades do trato urinário tem pouco efeito no feto dentro do útero. Mesmo malformações letais para os neonatos não comprometem os fetos, uma vez que a placenta e a mãe administram a função homeostática renal (5). Entretanto algumas delas fazem parte de anormalidades multissistêmicas complexas cujos efeitos cumulativos são letais no período fetal (18). No período perinatal, as anormalidades congênitas dos rins e do trato urinário são de principal importância, enquanto lesões específicas adquiridas e tumores são comparativamente raros (11).

A incidência de malformações renais em séries de necropsias perinatais é de aproximadamente 10\% (18). Muitas das anomalias renais e urinárias predispõem os rins a pielonefrite, hipertensão e litíase secundárias (21). Segundo Verma et al. (20), as malformações congênitas do trato genitourinário foram mais comuns entre o sexo masculino. Tan et al., em estudo de necropsias pediátricas, encontraram 2,5\% de malformações urinárias. Destas malformações, $35,9 \%$ eram casos de hidronefrose e/ou hidroureter; $29,5 \%$ de distúrbios císticos; $26,9 \%$ de agenesia renal; $11,5 \%$ de rins em ferradura; $5,1 \%$ de hipoplasia renal; e 1,3\% de rins pélvicos (19).

Ranadive et al. encontraram $1,8 \%$ de anomalias significativas do trato urinário que levaram à morte, com predominância masculina. Obstrução intra-uterina do trato urinário foi o principal fator etiológico que levou a alterações displásicas no parênquima renal (16). Neste estudo, com casos de necropsia, a prevalência de MFAU foi de $2,9 \%$, e, apesar dos dados encontrados na literatura, a análise comparativa entre os sexos em relação aos casos estudados mostrou que não existe diferença estatisticamente significativa entre o número de casos masculinos e femininos $(p<0,05)$, como mostra a Tabela 1.

Houve um maior número de RN e NM com MFAU em relação ao número total de casos, sendo $p<0,05$ nos dois grupos (RN e NM) (Tabela 4), e também em relação ao número geral de malformações, sendo que a análise estatística revelou significância somente no grupo RN. Isto reflete uma maior prevalência de MFAU nestes grupos etários, semelhante aos dados observados na literatura.

Observou-se um grande número de casos de NM no banco geral em relação ao grupo das malformações gerais $(p<0,05)$, como mostra a Tabela 4 . Isto reflete uma diminuição de casos de NM com malformações, proporção esta que se repete quando se analisam as malformações do aparelho urinário.

As subclasses de malformações mais prevalentes neste estudo foram A1, A2 e A3. A grande maioria dos casos pertencentes à subclasse $\mathrm{A} 1$ (anormalidades de massa renal) possuía agenesia renal unilateral (21 casos) ou bilateral (13 casos). A condição de agenesia renal em que o rim e o ureter estão completamente ausentes é devida a uma falha no desenvolvimento do ramo uretérico derivado do broto mesonéfrico ou a sua degeneração precoce (11). A agenesia renal é incompatível com a vida: a morte ocorre intra-útero ou no período neonatal devido a insuficiência respiratória (11). Algumas crianças com doenças renais menos severas podem manter função urinária intra-útero suficiente, de modo que a maturidade pulmonar é alcançada e anormalidades estruturais renais não são identificadas a não ser num período mais tardio da vida (18). A agenesia renal bilateral possui incidência de 1:4.000-6.000 nascimentos, e a unilateral e mais comum, 1:1000. Nos casos de agenesia 
Proporção do número de casos nos differentes grupos etários em relacão ao número total de Tabela 4 casos, ao número total de malformações e ao número de malformações do aparelho urinário

\begin{tabular}{lccccc}
\hline & Banco geral & MF gerais & MFAU & $p 1$ & p2 \\
NM & $33 \%$ & $16 \%$ & $14 \%$ & $\mathrm{p}<0,05$ & NS \\
RN & $35 \%$ & $44 \%$ & $55 \%$ & $\mathrm{p}<0,05$ & $\mathrm{p}<0,05$ \\
L & $16 \%$ & $23 \%$ & $18 \%$ & NS & NS \\
PE & $10 \%$ & $11 \%$ & $8 \%$ & NS & NS \\
E & $6 \%$ & $6 \%$ & $5 \%$ & NS & NS
\end{tabular}

RN: recém-natos; NM: natimortos; L: lactentes; PE: pré-escolares; E: escolares; p1: comparação estatística entre o número de casos do banco geral e de casos de malformações do aparelho urinário; p2: comparação estatística entre o número de casos de malformações gerais e de casos de malformações do aparelho urinário NS: valores sem diferença estatística significativa $(p>0,05)$.

bilateral, é muito comum a presença de malformações associadas do trato genital $(2,18)$. Neste estudo, a prevalência de agenesia renal (uni e bilateral) foi de $18,68 \%$ das MFAU. As anormalidades urológicas associadas a agenesia renal unilateral mais encontradas em estudo feito por Cascio et al. incluíam refluxo vesicoureteral primário (28\%), obstrução da junção vesicoureteral (11\%) e obstrução da junção ureteropélvica e ureterovesical (2\%), totalizando $48 \%$ de casos com tais malformações associadas (4).

Um ou mais cistos renais podem ser encontrados em necropsias em mais de $50 \%$ dos pacientes acima da idade de 50 anos. Sua comparativa raridade em adultos jovens e crianças sustentam o conceito de que eles são lesões adquiridas (3).

Malformações da bexiga são freqüentemente acompanhadas por anomalias no trato genital masculino e feminino, devido à inter-relação no desenvolvimento embriológico destes sistemas (18).

Segundo trabalho publicado por Isaksen et al., o diagnóstico pré-natal de anormalidades do aparelho urinário foi realizado em $27 \%$ dos casos que possuíam anomalias congênitas. Em $87 \%$ dos casos havia completa correlação entre as observações ultra-sonográficas e os achados de necropsia (8). A prevalência de malformações urinárias ao nascimento têm aumentado, provavelmente devido à disponibilidade de melhores técnicas diagnósticas $(7,9,14)$.

\section{Conclusão}

$\mathrm{Na}$ análise de 40 anos de necropsias da Unidade de Patologia Pediátrica e Perinatal do Serviço de Anatomia Patológica do Hospital de Clínicas de Curitiba, UFPR, observou-se que $2,9 \%$ dos casos apresentavam malformações urinárias, embora a literatura relate uma média que varia de $1,8 \%$ até $10 \%$ de malformações urinárias, dependendo do tipo de estudo.

A distribuição das malformações do aparelho urinário foi praticamente a mesma entre os sexos feminino (46\%) e masculino (54\%), embora a literatura relate uma maior predominância no sexo masculino.

O grupo etário mais acometido pelas MFAU é o dos recém-natos, concordando com os dados de literatura que afirmam que as alterações das funções homeostáticas renais causadas pelas malformações renais, mesmo que estas sejam letais, são supridas intra-útero pela placenta e pela mãe.

De uma maneira geral, as malformações do apareIho urinário mais encontradas na população avaliada foram as malformações renais e de trato urinário superior (150 casos), e, entre estas, as mais comuns foram as que englobam os casos de duplicação ureteral e da pelve renal e casos de obstrução, concordando com a literatura revisada.

\section{Referências}

1. Behrman, R.E.; Kliegman, R.M. \& Harbin, A.M. Tratado de Pediatria. 15. ed. Rio de Janeiro: Guanabara Koogan, 1996.

2. Berny, U. et al. Evaluation of the urinary tract in children with renal agenesis. Pol. M erkuriusz Lek., 2 (10): 259-61, 1997.

3. Berry, C. Paediatric pathology. 3. ed. Londres: Springer-Verlag Limited, 1995.
4. Cascio, S.; Paran, S. \& Puri, P.Associated urological anomalies in children with unilateral renal agenesis. J. Urolol., 162(3Pt2): 1081-3, 1999.

5. Dimmick, J.E. Developmental Pathology of the Embryio and Fetus. Cap 19. Philadelphia:J.B. Lippincott C ompany, 1992.

6. G ilbert-Barness, E. Potters Pathology of the fetus and infant. 3. ed Missouri: Mosby, 1997. 
7. Gunn,T.R.; M ora, J.D. \& Pease, P. A ntenatal diagnosis of urinary tract abnormalities by ultrasonography after 28 week's gestation: incidence and outcome. Am. J. Obstet. Gynecol., 172(2Pt1): 479-86,1995.

8. Isaksen, C.V. et al. Fetuses and infants with congenital urinary system anomalies: correlation between prenatal ultrasound and postmortem findings. Ultrasound Obstet. Gynecol., 15(3): 177-85, 2000.

9. James, C .A. et al.A ntenatally detected urinary tract abnormalities: changing incidence and management. Eur.J. Pediatr., 157(6): 508-11, 1998.

10. Kallen, K. Maternal smoking and urinary organ malformations. Int. J. Epidemiol., 26(3): 571-4, 1997.

11. Keeling,J.W . Fetal and N eonatal Pathology. Springer-Verlag, 1997.

12. Li, D.K. et al. Maternal smoking during pregnancy and the risk of congenital urinary tract anomalies. Am. J. Public Health, 86(2): 249-53, 1996.

13. Mishra,P.C :;Baveja, R.C ongenital malformations in the newborn: a prospective study. Indian Pediatr., 26: 32-6, 1989.

14. N azer, J.; Fernandez,P. \& Silva, C. U rinary tract malformations in newborns at the Clinical Maternity Hospital of the U niversity of C hile. Ver. Med. Chil., 126(12): 1472-7, 1998.

15. O dewolo, E.O .O et al. Pattern of congenital malformation in N igerian children. Int. Surg., 76:43-8, 1991.

16. Ranadive, $N$.U. et al. A uto psy study of infant kidneys in cases of urinary tract anomalies. Indian J. Pathol. M icrobiol., 39(3): 252, 1996.

17. Saller, D.N . et al. The clinical utility of the perinatal autopsy. JAM A, 273: 663-5, 1995.

18. Stocker, T. Pediatric Pathology. Philadelphia: J.B. Lippincott Company, 1992.

19. Tan, P.H .; C hiang, G.S. \& Tay, H. Pathology of urinary tract malformations in a paediatric autopsy series. Ann. Acad. Med. Singapore, 23(6): 838-43, 1994.

20.Verma, M .; C hhatwal, J. \& Singh, D. C ongenital malformation: retrospective study of 10,000 cases. Indian J. Pediatr., 58: 245-52, 1991.

21.W igglesworth,J.S. \& Singer, D.B. Textbook of fetal and perinatal pathology. 2 ed. Blackwell Science, 1998.

22. W oolf, A.S. A molecular and genetic view of human renal and urinary tract malformation. Kidney Int., 58(2):500-12, 2000. 